\title{
ANALYSIS OF ORDER OF STRESS SINGULARITY AT A VERTEX IN 3D TRANSVERSELY ISOTROPIC PIEZOELECTRIC DISSIMILAR BONDED JOINTS
}

\author{
Md. Shahidul Islam*, Md. Golam Kader, M. M. Kamal Uddin, and Mohiuddin Ahmed \\ Department of Mechanical Engineering, Khulna University of Engineering \& Technology, Khulna \\ "Corresponding e-mail: shahidulbitk@gmail.com
}

\begin{abstract}
The order of singularity near the vertex of bonded joints is one of the main factors responsible for debonding under mechanical or thermal loading. The distribution of stress singularity field near the vertex of bonded joints is very important to maintain the reliability of intelligent materials. In this paper, order of stress singularity at vertex in $3 D$ transversely isotropic piezoelectric dissimilar bonded joints is analyzed. Eigen analysis based on FEM is used for stress singularity field analysis of piezoelectric bonded joints. The eigen equation is used for calculating the order of stress singularity, and the angular function. The numerical result shows that the angular functions have large value near the interface edge than the inner portion of the joint. Therefore, there is a possibility to debond and delamination may occur at the interface edge of the piezoelectric bonded joints due to the higher stress and electric displacement concentration at the free edge.
\end{abstract}

Keywords: Piezoelectric Bonded Joints, Order of Singularity, Transversely Isotropic Material, Smart Structures, Eigen Analysis.

\section{INTRODUCTION}

Piezoelectric materials are being widely used in the electronics industry due to their high functionality. Mechanical stress occurs in piezoelectric material for any electric input. The stress concentrations caused by mechanical or electric loads may lead to crack initiation and extension, and sometimes the stress concentrations may be high enough to fracture the material parts. In the case of multilayer piezoelectric stacks, the electrodes that terminate inside the material body are a source of electric field, which can result in high stress concentrations. Reliable service lifetime predictions of piezoelectric components demand a complete understanding of the debonding processes of these materials. Industrial products such as electronic devices and heat endurance parts are composed of dissimilar materials. A mismatch of material properties causes a failure at the free edge of joint, because a stress concentration occurs along the free edge of interface especially at the vertex of joints ${ }^{1}$.

Sosa has suggested a general method of solving plane problems of piezoelectric media with defects ${ }^{2}$. Zak and Williams used eigen functions for analyzing stress singularity field at a crack tip perpendicular to a bimaterial interface. They found that a real part of eigen value is within the range of 0 to 1 , and expressed a relationship between stress distribution and the order of stress singularity at the crack tip ${ }^{3}$. Aksentian determined eigen values and eigen vectors at the singular point in plane intersecting a free edge of the interface in three dimensional dissimilar joints ${ }^{4}$. Yamada and Okumura developed a finite element analysis for solving eigen value equation to determine directly the order of stress singularity and the angular variation of the stress and displacement fields ${ }^{5}$. Pageau and Biggers determined the order of stress singularity and the angular variation of the displacement and the stress fields around the singular points in plane intersecting a wedge front in the three-dimensional anisotropic material structures using the two dimensional displacement formulation under a plane strain assumption ${ }^{6}$.

Thus, at present, no clear picture exits of the effects of stress singularity field near the vertex of three-dimensional transversely isotropic piezoelectric bimaterial joints. Therefore, the purpose of this analysis is to obtain a better understanding of the effect of stress singularity field near the vertex of transversely isotropic piezoelectric bimaterial joints on interface. In this study, three-dimensional FEM eigen equation was formulated for analyzing the order of singularity in piezoelectric bimaterial joints.

\section{FORMULA OF ANALYSIS}

In the absence of body forces and free charges, the equilibrium equations of piezoelectric materials are expressed as follows ${ }^{7}$ :

$$
\begin{gathered}
\sigma_{i j, j}=0 \\
d_{i, i}=0
\end{gathered}
$$

where $\sigma_{i j}$ represents the stress and di the electric displacement.

The constitutive relations are shown as follows:

$$
\begin{aligned}
& \sigma_{i j}=c_{i j k l} \varepsilon_{k l}-e_{k i j} E_{k} \\
& d_{i}=e_{i k l} \varepsilon_{k l}+\chi_{i k} E_{k}
\end{aligned}
$$

where $c_{i j k l}$ is the elastic constant, $e_{i k l}$ and $\chi_{i k}$ are the piezoelectric constant and electric permittivity (dielectric constant), respectively. 
The elastic strain-displacement and electric field-potential relations are presented as follows:

$$
\begin{gathered}
\varepsilon_{i j}=\frac{1}{2}\left(u_{j, i}+u_{i, j}\right) \\
E_{i}=-\psi_{, i}
\end{gathered}
$$

where $\varepsilon_{i j}$ is the strain, and Ei is the eclectic field. ui is the elastic displacement and $\psi$ is the electric potential.

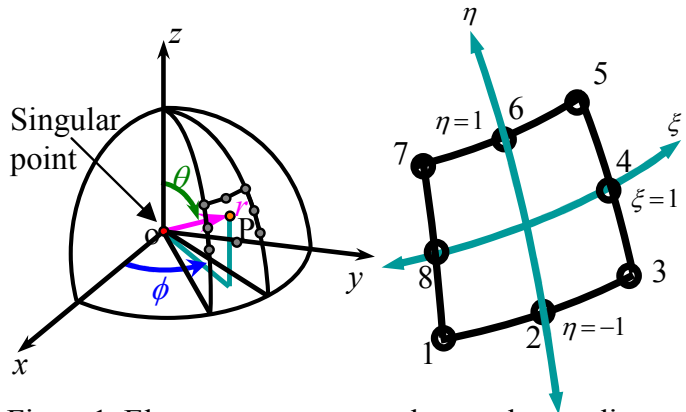

Figure1. Element geometry and natural co-ordinates at a free edge singular point

Figure 1. represents the geometry of a typical case where a singular stress state occurs at the point $o$. The region surrounding the singular point is divided into a number of quadratic pyramidal elements with a summit $o$, with each element being located in spherical co-ordinates $r, \theta$, and $\phi$ by its nodes 1 to 8 . A point $P$ in the element can be located using the singular transformation by the relations.

$$
\begin{gathered}
r=r_{o}\left(\frac{1+\alpha}{2}\right)^{1 / P} \text { or, } \rho=\frac{r}{r_{o}}\left(\frac{1+\alpha}{2}\right)^{1 / P} \\
\theta=\sum_{i=1}^{8} H_{i} \theta_{i} \quad \text { and } \quad \phi=\sum_{i=1}^{8} H_{i} \phi_{i}
\end{gathered}
$$

where,

$$
\begin{array}{ll}
H_{1}=-\frac{1}{4}(1-\eta)(1-\xi)(\eta+\xi+1) & H_{2}=\frac{1}{2}(1-\eta)\left(1-\xi^{2}\right) \\
H_{3}=-\frac{1}{4}(1-\eta)(1+\xi)(-\eta+\xi-1) & H_{4}=\frac{1}{2}\left(1-\eta^{2}\right)(1+\xi) \\
H_{5}=\frac{1}{4}(1+\eta)(1+\xi)(\eta+\xi-1) & H_{6}=\frac{1}{2}(1+\eta)\left(1-\xi^{2}\right) \\
H_{7}=\frac{1}{4}(1+\eta)(1-\xi)(\eta-\xi-1) & H_{8}=\frac{1}{2}\left(1-\eta^{2}\right)(1-\xi)
\end{array}
$$

$\theta$ and $\phi$ are the nodal values of the angular co-ordinates and $\alpha, \eta$, and $\xi$ are natural co-ordinates of the element whose ranges are shown in Fig.1. $H$ interpolation function, $p$ eigen value, $\rho=r / r_{o}, r$ the distance from the singular point. The elastic displacement and electric potential relations are:

$$
\begin{aligned}
& \left(\bar{u}-\bar{u}_{o}\right)=\left(\frac{1+\alpha}{2}\right)\left[\sum_{i=1}^{8} H_{i}\left(\bar{u}_{i}-\bar{u}_{o}\right)\right] \\
& \left(\bar{\psi}-\bar{\psi}_{o}\right)=\left(\frac{1+\alpha}{2}\right)\left[\sum_{i=1}^{8} H_{i}\left(\bar{\psi}_{i}-\bar{\psi}_{o}\right)\right]
\end{aligned}
$$

where $\bar{u}_{o}$ and $\bar{u}$ is the elastic displacement at $o$ and $P$, respectively, and $\bar{\psi}_{o}$ and $\bar{\psi}$ is the electric potential at $o$ and $P$, respectively.

$$
\begin{array}{cl}
u=\left(\bar{u}-\bar{u}_{o}\right), & u_{i}=\left(\bar{u}_{i}-\bar{u}_{o}\right) \\
\psi=\left(\bar{\psi}-\bar{\psi}_{o}\right), & \psi_{i}=\left(\bar{\psi}_{i}-\bar{\psi}_{o}\right)
\end{array}
$$

From the above equation, the elastic displacement and electric potential relations are expressed as follows:

$$
\begin{gathered}
u_{k}=\rho^{p}\left[\sum_{i=1}^{8} H_{i} u_{k i}\right] \quad(k=r, \theta, \phi) \\
\psi=\rho^{p}\left[\sum_{i=1}^{8} H_{i} \psi_{i}\right]
\end{gathered}
$$

\section{Problem DESCRIPTION \& NUMERICAL EXAMPLE}

For a transversely isotropic piezoelectric material, taking $z$ to be parallel to the poling axis of the material, by convention, the constitutive relation is expressed in the following form:

$$
\{\sigma\}=[c]\{\varepsilon\}-[e]\{E\} \quad \text { and } \quad\{d\}=[e]^{T}\{\varepsilon\}+[\chi]\{E\}
$$

where $\{\sigma\}$ and $\{\varepsilon\}$ are the stress and strain wnich are the mechanical field variables, $\{d\}$ and $\{E\}$ are the electric displacement and electric field, respectively, $[c]$ is the elastic constant, and $[e]$ and $[\chi]$ are the piezoelectric constant and electric permittivity (dielectric constant) respectively.

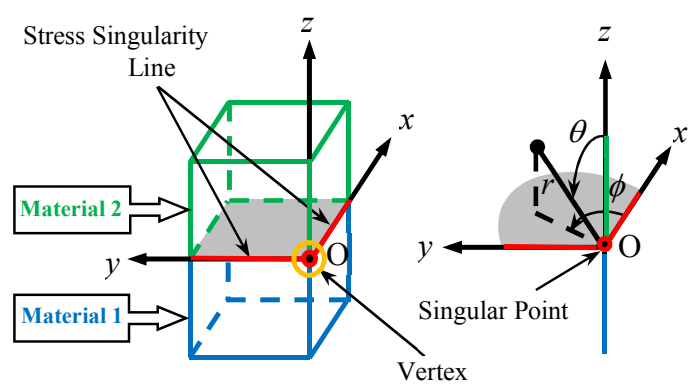

Figure 2. Singular point of 3D piezoelectric joint in $x, y, z$ plane.

The eigen equation is formulated for determining the order of stress singularity as follows ${ }^{8}$.

$$
\left(p^{2}[\mathbf{A}]+p[\mathbf{B}]+[\mathbf{C}]\right)\{\mathbf{U}\}=\{\mathbf{0}\}
$$

Where $p$ represents the characteristic root, which is related to the order of singularity, $\lambda$, as $\lambda=1-p$. $[\boldsymbol{A}]$, $[\boldsymbol{B}]$ and $[\boldsymbol{C}]$ are matrices composed of material properties, and $\{\boldsymbol{U}\}$ represents the elastic displacement and electric potential vector. 
In the present paper, the order of singularity is investigated varying the material constants. A ratio of material constants to a specified material constant is introduced as follows? .

$$
\frac{e}{e_{n}}=s \text { and } \frac{\chi}{\chi_{n}}=s
$$

Where $e_{n}$ and $\chi_{n}$ represent the referential piezoelectric and dielectric constants of PZT-5H and PZT-5A. $e$ and $\chi$ represent the new piezoelectric and dielectric constants. The value of $S$ varies from 0.0001 to 10000 .

The elastic displacement and electric potential equation is expressed by the following equitation.

$$
\begin{gathered}
u_{j}(r, \theta, \phi)=b_{j}(\theta, \phi) r^{1-\lambda} \\
\psi(r, \theta, \phi)=q(\theta, \phi) r^{1-\lambda}
\end{gathered}
$$

By differentiating the above two equations, get the angular function of strain and electric field equation respectively. The stress and electric displacement distribution equations in the stress singularity region can be expressed as follows.

$$
\begin{aligned}
& \sigma_{i j}(r, \theta, \phi)=K_{i j} r^{-\lambda} f_{i j}(\theta, \phi) \\
& d_{i}(r, \theta, \phi)=F_{i} r^{-\lambda} l_{i}(\theta, \phi)
\end{aligned}
$$

Where $r$ represents the distance from the stress singular point, $b_{j}(\theta, \phi)$ the angular function of elastic displacement, $q(\theta, \phi)$ the angular function of electric potential, $f_{i j}(\theta, \phi)$ the angular function of stress distribution, $l_{i}(\theta, \phi)$ the angular function of electric displacement, $K_{i j}$ the intensity of singularity, $F_{i}$ the intensity of electric field, and $\lambda$ the order of stress singularity. Angular functions of stress components obtained from eigen analysis in Eq. (15). are examined.

Table 1. Material properties of piezoelectric materials

\begin{tabular}{|c|c|c|c|c|c|}
\hline \multirow{2}{*}{ Material } & \multicolumn{5}{|c|}{ Elastic Constant, $10^{10} \mathrm{~N} / \mathrm{m}^{2}$} \\
\cline { 2 - 6 } & $\mathrm{c}_{11}$ & $\mathrm{c}_{12}$ & $\mathrm{c}_{13}$ & $\mathrm{c}_{33}$ & $\mathrm{c}_{44}$ \\
\hline PZT-5H & 12.6 & 5.50 & 5.30 & 11.7 & 3.53 \\
\hline PZT-5A & 12.1 & 7.54 & 7.52 & 11.3 & 2.11 \\
\hline PZT-A & 168 & 163 & 27.1 & 60.0 & 60.0 \\
\hline PZT-B & 168 & 163 & 27.1 & 60.0 & 60.0 \\
\hline
\end{tabular}

\begin{tabular}{|c|c|c|c|c|c|}
\hline \multirow{3}{*}{ Material } & \multicolumn{3}{|c|}{$\begin{array}{c}\text { Piezoelectric } \\
\text { Constant, C/m }\end{array}$} & \multicolumn{2}{c|}{$\begin{array}{c}\text { Dielectric } \\
\text { Constant, } \\
10^{-10} \text { C/Vm }\end{array}$} \\
\cline { 2 - 6 } & $\mathrm{e}_{15}$ & $\mathrm{e}_{31}$ & $\mathrm{e}_{33}$ & $\chi_{11}$ & $\chi_{33}$ \\
\hline PZT-5H & 17.0 & -6.50 & 23.3 & 151.0 & 130.0 \\
\hline PZT-5A & 12.3 & -5.20 & 15.8 & 81.1 & 73.5 \\
\hline PZT-A & -0.9 & 7.1 & 4.6 & 36.0 & 34.0 \\
\hline PZT-A & -14.0 & 36 & 43 & 200 & 247 \\
\hline
\end{tabular}

In this analysis, first of all, eigen values and eigen vectors are investigated by eigen analysis when two different materials are bonded. The order of singularity $\lambda$, at the vertex for the model shown in Fig. 2 is calculated. Solving eigen equation yields many roots $\mathrm{p}$ and eigen vectors corresponding to each eigen value are obtained. However, if the root $\mathrm{p}$ is within the range of $0<p<1$, this fact indicates that the stress field has singularity. The value of $\lambda_{\text {line }}$ is compared with $\lambda_{2 D}$ which was obtained by Xu et al. for 2D joint. The model of joint has the same shape and material properties as that used in their paper13. Material properties are shown in Table 1 . $\lambda_{\text {line }}$ obtained by the present eigen analysis is 0.01272 and $\lambda_{2 \mathrm{D}}$ is 0.01276 , two values obtained from different methods are almost agreed with each other. The value of order of singularity at the singularity corner is 0.0352 .

The effect of material constants on the order of stress singularity is determined with the help of Eq. (16) and graph is shown below. Fig. 3 shows the variation of order of singularity by varying the piezoelectric, dielectric and both piezoelectric and dielectric constants of PZT-5H and PZT-5A respectively. The order of singularity for variation of piezoelectric and piezo \& dielectric constant shows almost same nature, but for dielectric constant shows the opposite nature. It is found from the figure that the variation of material constants from 0.01 to 100 alter the order of stress singularity more significantly.

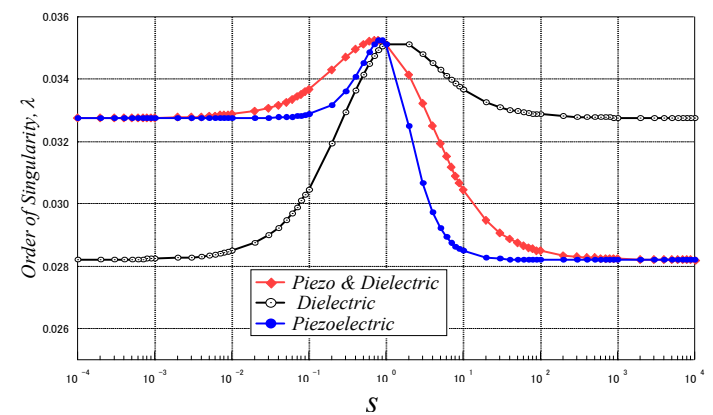

Figure 3. Variation of order of singularity against material constant for PZT-5H and PZT-5A.

The distributions of elastic displacement and electric potential are shown in Fig. 4. It is found that, the angular function of elastic displacement and electric potential is continuous at the interface of the joints and has higher value at the free edge than the inner portion. So there is a possibility to debond near the free edge of the joint. It is also found that the angular function of elastic displacement and electric potential at $\phi=0^{\circ}$ is agreed with that at $\phi=90^{\circ}$. The figure of $b_{\theta}$ and $b_{r}$ is symmetry with respect to $\phi$ and the figure of $b_{\phi}$ and $q$ is antisymmetry with respect to $\phi$. The distributions of normalized angular function of strain and electric field with respect to the angle $\phi$ at $\theta=90^{\circ}$ in the singular field for the piezoelectric bonded structure 

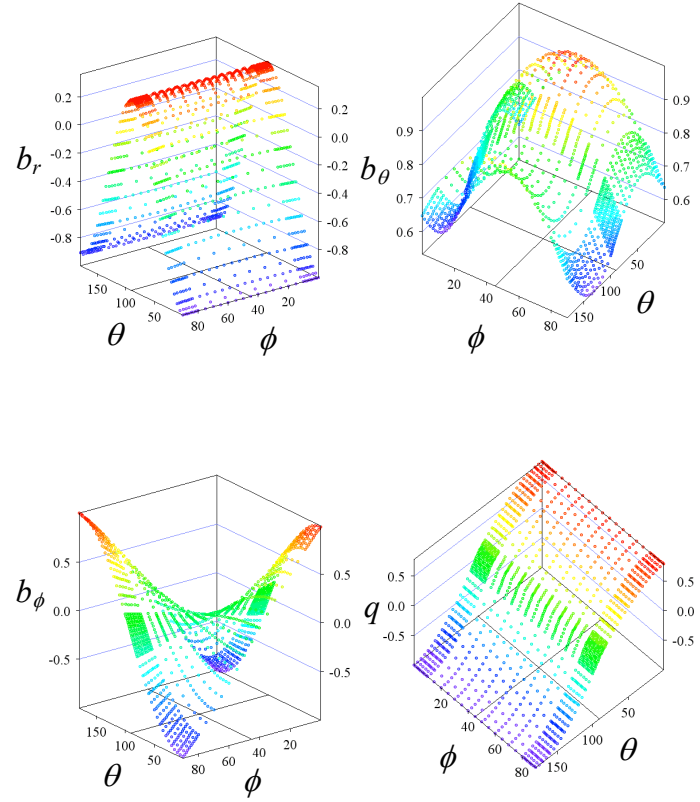

Figure 4. Distribution of $b_{i} \& q$ against $\phi$ and $\theta$.

are shown in Figs. 5 and 6 . The angular function of strain $t_{r r}, t_{r \theta}, t_{\theta \theta}$ and $t_{\phi \phi}$ are symmetry with respect to angle $\phi$ at $45^{\circ}$ but $t_{r \phi}$ and $t_{\phi \theta}$ are anti-symmetry with respect to angle $\phi$ at $45^{\circ}$. Similarly the angular function of electric fields $e_{r}$ and $e_{\theta}$ are symmetry with respect to angle $\phi$ at $45^{\circ}$ but $e_{\phi}$ is anti-symmetry with respect to angle $\phi$ at $45^{\circ}$. It is also shown from the figure that the strain and electric field have larger value near the free edge of the bonded joints.

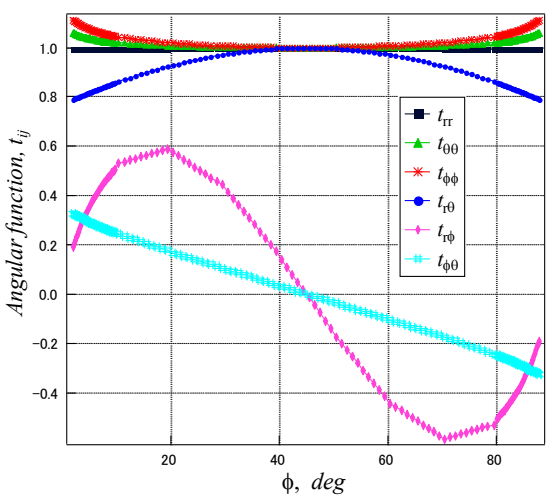

Figure 5. Distribution of normalized angular function of strain against $\phi$ at $\theta=90^{\circ}$.

The distributions of normalized angular function of stress and electric displacement with respect to the angle $\phi$ at $\theta=90^{\circ}$ for the piezoelectric bonded structure are shown in Figs. 7 and 8. These distributions are nearly similar to the distribution of angular function strain and electric field. The angular function of stress $f_{\theta \theta}$ and $f_{r \theta}$ are symmetry with respect to angle $\phi$ at $45^{\circ}$ but $f_{\phi \theta}$, is anti-symmetry with respect to angle $\phi$ at $45^{\circ}$. Similarly the angular function of electric displacement $d_{r}$, and $d_{\theta}$, are symmetry with respect to angle $\phi$ at $45^{\circ}$ but $d_{\phi}$ is anti-symmetry with respect to angle $\phi$ at $45^{\circ}$. It is also shown from the figure that the stress and electric displacement have larger value near the free edge of the bonded joints. So there is another possibility to debond and delamination occurs near the free edge of the bonded joints.

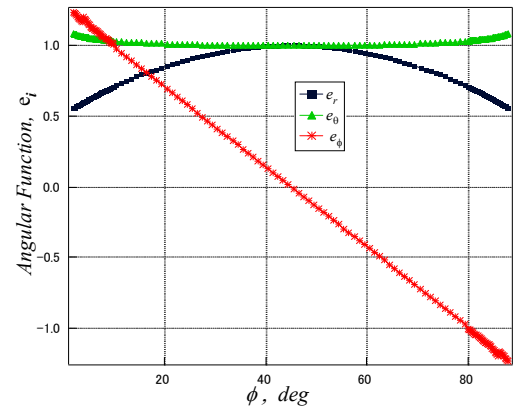

Figure 6. Distribution of normalized angular function of electric field against $\phi$ at $\theta=90^{\circ}$.

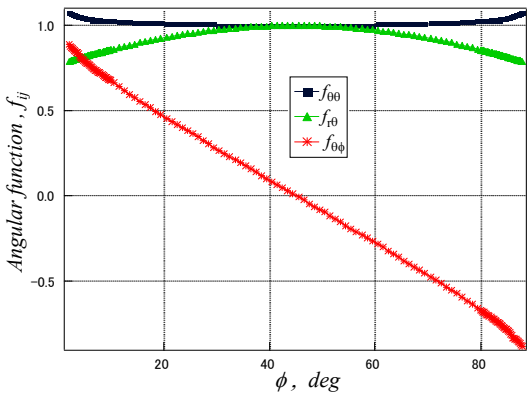

Figure 7. Distribution of normalized angular function of stress against $\phi$ at $\theta=90^{\circ}$.

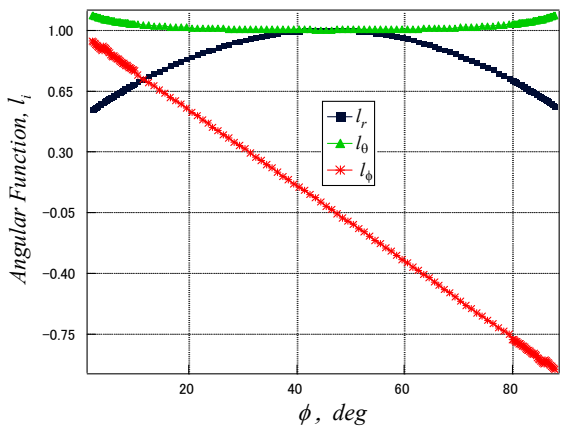

Figure 8. Distribution of normalized angular function of electric displacement against $\phi$ at $\theta=$ $90^{\circ}$. 


\section{CONCLUSION}

In this paper, a finite element method formulation near the vertex of transversely isotropic piezoelectric bimaterial joint was presented. Angular functions for singularities corner were derived from eigen analysis using a finite element method. From the numerical result, the following conclusions can be drawn for the piezoelectric bimaterial joints.

a) Change of material constant of piezoelectric material in-between the range from 0.01 to 100 affects the order of stress singularity more significantly.

b) The higher angular function occurs at the free edge of the material joint than the inner portion of the joint.

c) There is a possibility to debond and delamination may occur at the interface edge of the piezoelectric bonded joints due to the higher stress concentration at the free edge.

\section{REFERENCES}

1. Attaporn W., and Koguchi H. , 2009, "Intensity of stress singularity at a vertex and along the free edges of the interface in 3D-dissimilar material joints using 3D-enriched FEM", CMES, 39(3), pp. 237-262.

2. Sosa H. A., 1991, "Plane problems in piezoelectric media with defects", Int. J.of Solids Structure, 28, pp.491-505.

3. Zak A. R., and Williams M. L., 1963, "Crack point stress singularities at a bi-material interface", J. of Applied Mechanics, Brief Notes, pp.142-143.

4. Aksentian O. K., 1967, "Singularities of the stress-strain state of a plate in the neighborhood of an edge”, PMM, 31(1), pp.178-186.
5. Yamada Y., and Okumura H., 1981, "Analysis of local stress in composite materials by 3-D finite element", Proc. of the Japan-U.S. Conference, Tokyo, pp. 55-64.

6. Pageau S. S., and Biggers Jr. S. B., 1996, “A finite element approach to three-dimensional singular stress states in anisotropic multi-material wedges and junctions", Int. J. of Solids Structure, 33, pp. 33-47.

7. Liew K. M., and Liang J., 2002, "Modeling of 3D piezoelectric and elastic bimaterials using the boundary element method", Computational Mechanics, 29, pp. 151-162.

8. Pageau S. S., Joseph P. F., and Biggers, Jr. S. B., 1995, "Finite element analysis of anisotropic materials with singular inplane stress fields," Int. J.of Solids Structure, 32, pp. 571-591.

9. Islam M. S., and Koguchi H., 2010, "Characteristics of singular stress distribution at a vertex in transversely isotropic piezoelectric dissimilar material joints," JSME J. of solid Mechanics and Material Engineering, 4(7), pp. 1011-1026.

10. XU J. Q. and Mutoh Y., 2001, "Singularity at the interface edge of bonded transversely isotropic piezoelectric dissimilar material," JSME Int. J., Series A, 44(4), pp.556-566.

11. Koguchi H. and Fujimagari M., 2001, Analysis for the Order of Stress Singularity, Displacement and Stress Field at a Vertex of Three-Dimensional Joints in Electric Devices, Transactions of the Japan Society of Mechanical Engineers, Series A, 67, pp.1-8. 